\title{
Teleconsent - A New Modality for Informed Consenting
}

\author{
Saif Khairat ${ }^{1 *}$ and Jihad S. Obeid ${ }^{2}$ \\ ${ }^{1}$ Carolina Health Informatics Program and School of Nursing, University of North Carolina, Chapel Hill, North Carolina, USA \\ ${ }^{2}$ Department of Public Health Sciences, Medical University of South Carolina, Charleston, South Carolina, USA
}

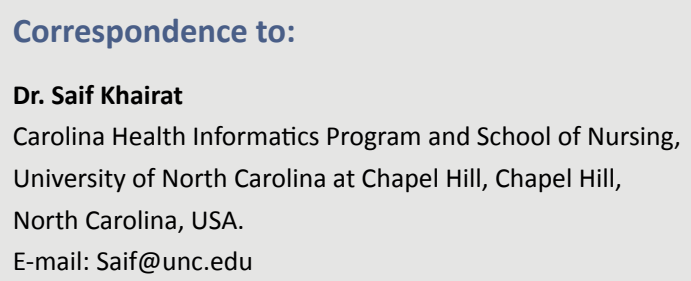

\section{EJBI 2018; 14(4):63-64}

Received: July 23, 2018

Accepted: August 17, 2018

Published: August 24, 2018

Obtaining informed consent, the process wherein the be as cognitively attentive relative to the traditional face-to-face research participant or patient formally authorizes the researcher interaction when the two parties are in one room speaking to or provider to provide care or to exchange medical information each other. The environment is controlled and distractions are between providers, is necessary for the delivery of care or minimal. Teleconsent ensures a similar controlled environment conducting research. The introduction of health information with both parties seeing each other live and therefore warrants technologies such as telehealth, the ability to deliver healthcare the full attention of both parties.

virtually, has facilitated accessibility levels to healthcare services. We introduce a new modality of telehealth to obtain the consent of patients and providers using information technologies. Teleconsent differs from traditional informed consent by extricating the in-person and pen-to-paper signature, instead having a video interaction between the participant and the provider followed by a photo-based or handwritten e-signature.

The differences between teleconsent and other consent modalities are distinct. The standard for truly informational consent process remains a face-to-face discussion with the patient. However, this is associated with travel costs and time, which is particularly burdensome in multi-site clinical trials or distant patient residence. For in-person consent to occur both parties need to be physically present in the same location at the same time, provide a clear explanation of the study protocol to the potential applicant, and obtain the applicants signature.

For e-consenting, which is the process of asynchronous consenting, a call occurs between both parties explaining the study resc protocol, and then a consent form is faxed, emailed, or mailed to the participant to sign and then, send it back to the research for coordinator. The requirement is for the participant to have access to a fax machine; or email, scanner, and a printer to be able to send and receive the consent form, which may not be available among rural and disadvantaged communities. Teleconsent overcomes the transportation and technological barriers by minimizing the requirements to a computer connected to the internet.

Another challenge with asynchronous consent modalities is

Opportunities to teleconsent do include better convenience to both the patient and provider; however, its true benefits lie largely in the improvement of patient-centered care. In contrast to asynchronous online consent, teleconsent allows providers to not only thoroughly explain what is to be consented, but also allows for follow-up questions and further explanation. Moreover, teleconsent does not require any transportation costs as compared to the in-person method, and affords significant time savings associated with commute and wait times. This is expected to improve patient satisfaction as well as provider efficacy. Previous innovative solutions such as e-consent, the process of faxing or emailing the consent form to be signed and returned by electronic means, lack the ability for the provider to synchronously interact with patients and answer their questions or concerns, which prolongs the consent process and may contribute to high retention rates.

There are of course barriers to teleconsent that need to be resolved. The most basic or obvious would be the addition of technology to a human practice; the culture of using IT for healthcare and research may not be mature. The internet connection may not be sufficient or not available at all in rural geographical areas. A poor connection would hinder sufficient communication of either or both parties and therefore, drawing out the process further leading to longer call duration, multiple calls, low quality video and audio feeds. Will culture and IT infrastructure play a role in the success or failure of teleconsent?

In conclusion, teleconsent is another facet of telehealth the lack of concentration of the participant or patient and the that has the potential to ameliorate global healthcare. Through provider or researcher. During a phone conversation or when teleconsent, providers and researchers can reach their patients digitally sending the consent forms, the participants may not and participants despite the time difference or location, which 
increases the chances of successfully obtaining the informed Acknowledgement consent and higher mutual satisfaction levels. Nevertheless, more research is needed in order to refine the process and guarantee that teleconsent will advance of the overall patient and provider experience without adversely impacting the informed consent process.

This work was supported by the National Center for Advancing Translational Sciences (NCATS), National Institutes of Health, through Grant Numbers R21 TR002088, UL1 TR001450, UL1TR002489, and 2KR981704.. 